\title{
Considerations for RFID-Based Indoor Simultaneous Tracking ${ }^{\star}$
}

\author{
Apostolia Papapostolou and Hakima Chaouchi \\ Telecom-Sudparis, CNRS SAMOVAR, UMR 5157, LOR department \\ \{apostolia.papapostolou, hakima. chaouchi\}@it-sudparis.eu
}

\begin{abstract}
Context-aware applications is not just a vision. Advances in wireless communications and mobile capabilities have revolutionized the way services are brought to users, i.e. adapted to their context. Location is a key attribute of the term context and thus, an accurate location determination system is of paramount importance. RFID (Radio Frequency IDentification) is an emerging technology and recently has been explored for its applicability in location sensing systems. In this paper, we focus on an RFID-based localization approach in an indoor multi-user environment and model its most adverse implicating factors, that is collisions among its main components and interference from indoor characteristics. Extensive simulations are conducted to characterize and evaluate the performance behavior of the proposed scheme in environments with different levels of severity.
\end{abstract}

Keywords: RFID, Location, Simultaneous Trucking, Reader Collisions.

The proliferation of wireless technologies, mobile computing and Internet has spurred the development of innovative context-aware services, with location being a crucial attribute of the general term context. Additionally, network functionalities such as mobility management, network planning, load planning etc, can be improved if location information is available. However, for accomplishing these visions, a location determination system which provides an accurate, reliable and fast estimation of users or devices position is required.

WLAN-based indoor location systems have attracted the interest of the research and industry communities. The main benefits of such systems is their low cost and ease of deployment since they rely on the existing WLAN infrastructure. Moreover, the number of WiFi-enabled devices is rapidly increasing in the markets. However, the achieved accuracy is limited.

RFID is a rapidly developing short range technology which uses wireless communication for automatic identification of objects. An RFID system consists of two main components, the tag and the reader. The RFID reader can read data emitted from the RFID tags within its reading range by using a defined Radio Frequency $(\mathrm{RF})$ and protocol. The RFID tags can be either passive or active.

\footnotetext{
* This paper is conducted in the French national research project ANR 2006 SUN:
}

Situated and Ubiquitous Networks. 
Passive tags operate without battery, they just backscatter (far-field case) the RF signal transmitted to them from a reader. Active tags contain both a radio transceiver and a battery. Thus, passive tags compared to active tags are less expensive and with unlimited lifetime but with reduced read range capability. Due to its relatively low cost and its technical capabilities, RFID is considered as an attractive technology for location sensing. Correlating tag ids with specific locations can aid determining the location of a reader based on the location information retrieved from the tags within its range. However, RFID technology has some limitations which should be considered before employing it for localization. Being a short-range communication technology requires a dense deployment of tags so that readers can always detect some of them. Additionally, interference from specific materials affect the read range of a reader. Last but not least the reader collision problem prevents the colliding readers from communicating with the RFID tags in their respective reading zones and thus the simultaneous tracking of multiple users is more complicated than the single-user case.

In this work, we address, model and evaluate the problem of tracking multiple reader-enabled users in the presence of interference and collisions among their readers when they simultaneously attempt to communicate their common tags.

The remainder of this paper is organized as follows. Section 1 outlines work related to the localization problem. In section 2 an overview of the RFID technology is provided. In section 3 the concept of localization relying on RFID and its limitations are introduced. In section 4 we model the system, communication properties and positioning methods of an RFID-based localization system. Simulations results in section 6 evaluate its performance and finally in section 7 we conclude our study.

\section{Related Work}

The most well known positioning system is the Global Positioning System (GPS), [1, which is satellite-based and is successful for tracking users in outdoor environments. However, the inability of satellites signals to penetrate buildings cause the complete failure of GPS indoors. For indoor location sensing a number of wireless technologies have been proposed, such as infrared, 7] ultrasound, 8], UWB, WiFi, [3]-6], Bluetooth, RFID, ZigBee and three are the main positioning techniques that are employed, namely triangulation, scene analysis and proximity. For triangulation methods, either the time of arrival (TOA), time difference of arrival (DTOA), angle of arrival (AOA) or strength (RSS) of the signals received from the surrounding transmitters are used for locating a user. Scene analysis methods require an offline phase for characterizing the radio behavior of the area under study. Proximity methods are based on the identification of objects with known location or topology constraints. 2] provides an interesting survey for positioning methods and schemes.

RFID-based schemes, such as [10-14, rely on the existence of RFID readers in the area and modify any of the three well-known positioning methods to locate 
users who are equipped with an RFID tag. Others, such as [12] and [15], rely on the deployment of tags in the area and try to locate a single user who is equipped with an RFID reader.

\section{RFID Technology Overview}

RFID (Radio Frequency IDentification) is an automatic identification system that consists of two basic hardware components, a tag and a reader. A tag has an identification (ID) stored in its memory that is represented by a bit string. The reader, which typically is a powerful device with memory and computational resources, is able to read the IDs of the tags in the neighborhood by running a simple link-layer protocol over the wireless channel. Various types of tags exist which differ significantly in their computational capabilities. They range from dump passive tags which operate without battery but they respond simply to reader's queries to smart active tags which contain both radio transceiver, memory and a power supply. Thus, passive tags compared to active tags are less expensive and with unlimited lifetime but with reduced read range capability. Due to their low cost, passive tags are anticipated to be a popular choice especially for large scale deployment.

The communication between a reader and a passive tag is done using either magnetic or electromagnetic coupling. Coupling is the transfer of energy from one medium to another medium, and tags use it to obtain power from the reader to transfer data. Two are the main types of coupling, inductive or backscatter, depending on whether the tags are operating in the near-field or far-field of the interrogator, respectively. A key difference between them is that far-field communication has a longer read range compared to near field communication.

RFID systems operate in the Industry, Scientific and Medical (ISM) frequency band that ranges from $100 \mathrm{KHz}$ to $5.8 \mathrm{GHz}$ but they are further subdivided into four categories according to their operating frequency: Low Frequency (LF), High Frequency (HF), Ultra-High Frequency (UHF) and Microwave. Tags operating at UHF and microwave frequencies use far-field and couple with the interrogator using backscatter. Recently, ultra-high frequency (UHF) band passive RFID systems have drawn a great deal of attention. In this paper, we consider RFID tags operating in the UHF (890-960 MHz) frequency band.

\section{RFID-Based Localization}

RFID is a rapidly developing short range technology which uses wireless communication for automatic identification of objects. Due to the low cost of passive tags, the non-LOS requirement, the simultaneous reading of multiple tags, and the reduced sensitivity regarding user orientation, the RFID is considered as an attractive technology for location sensing. 


\subsection{Methology Concept}

RFID-based positioning schemes can be classified into two general categories depending on the type of the RFID component supported by the target's device. For the first type we assume that the user device is equipped with a tag and a number of readers are placed in the area. In this case, any of the general positioning techniques, i.e. triangulation, scene analysis or proximity can be employed to estimate the location of the user. In the second case, the user's terminal is equipped with an RFID reader and passive or active tags with static and known coordinates are deployed in the area. Its location can be calculated based on the location information retrieved from the detected tags.

We focus on the second case and we attempt to determine the location of multiple reader-enabled user terminals. More precisely, passive tags are deployed in the floor of the areas such that a grid is formed and they are termed as reference tags.

\subsection{Limitations}

Even though RFID technology has promising key characteristics for location sensing, it has also some limitations which become more intense in the case of simultaneous tracking in a multi-user environment and thus should be taken into account before employing an RFID system for localization.

Since RFID technology uses electromagnetic waves for information exchange between tags and readers, how radio waves behave under various conditions in the RFID interrogation zone (IZ) affects the performance of the RFID system. Radio waves propagate from their source and reach the receiver. During their travel, they pass through different materials, encounter interference from their own reflection and from other signals, and may be absorbed or blocked by various objects in their path. The material of the object to which the tag is attached may change the property of the tag, even to the point it is not detected by its reader.

However, the most harmful type of interference is the one among its components which is known as the RFID collision problem. Three are its main types: tag collision, multiple reader-to-tag collision and reader-to-reader collision.

\section{- Tag Collisions}

This type of interference arises when multiple tags are simultaneously energized by the reader and reflect their respective signals back to the reader. Due to a mixture of scattered waves, the reader cannot differentiate individual IDs from the tags; therefore, anti-collision mechanisms such as those known as binary-tree and ALOHA are needed. For dealing with this type of interference the EPCglobal class-1 standard implements an algorithm based on a Query Tree protocol, [17. Adopting this anti-collision algorithm a reader can potentially read 500 collocated tags per second.

- Reader-to-Reader Collisions

Reader-to-reader collision is induced when a signal from one reader reaches other readers. This can happen even if there is no intersection among reader 


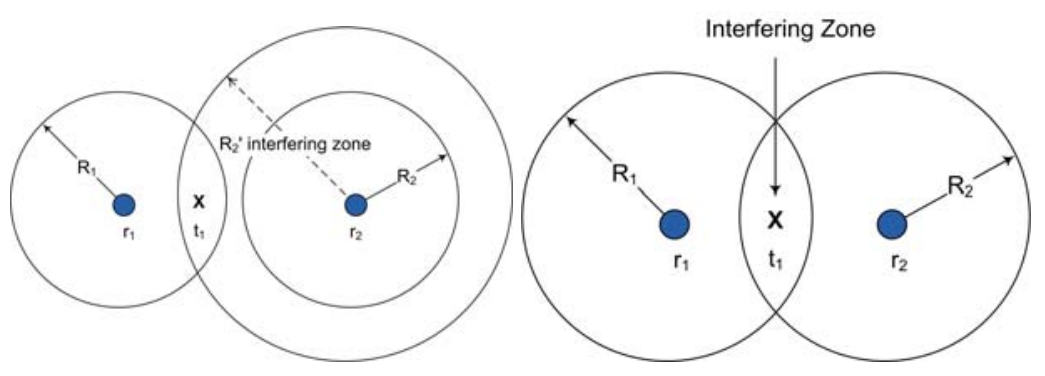

(a) Reader-to-Reader Collision

(b) Multiple Reader-to-Tag Collision

Fig. 1. The Collision Problem

interrogation ranges but because a neighbor reader's strong signal interferes with the weak reflected signal from a tag. Figure 1(a) demonstrates an example of collision from reader $r_{2}$ to reader $r_{1}$ when the latter tries to retrieve data from tag $t_{1}$. Generally, signal strength of a reader is superior to that of a tag and therefore if the frequency channel occupied by $r_{2}$ is the same as that between $t_{1}$ and $r_{1}, r_{1}$ is no longer able to listen to $t_{1} \mathrm{~s}$ response.

- Multiple Reader-to-Tag Collisions

Multiple reader-to-tag collision happens when a tag is located at the intersection of two or more reader interrogation ranges and the readers attempt to communicate with the tag simultaneously. As illustrated in figure $1(\mathrm{~b})$ when readers $r_{1}$ and $r_{2}$ transmit query messages to a tag $t_{1}, t_{1}$ might not be able to read the query messages from $r_{1}$ and $r_{2}$ due to interference.

Reviewing the current literature several scheduling-based solutions can be found. However, applying any of the general Multiple Access (MA) mechanisms, i.e. Time Division MA (TDMA), Frequency Division MA (FDMA), Code Division MA (CDMA) and Carrier Sense MA (CSMA), directly in RFID is not trivial, mainly due to the limited capabilities of the RFID passive tags.

\section{System and Communication Model}

In this section we try to derive a realistic mathematical model for describing the communication properties among the component of an RFID by considering their specifications and the main sources of error related to the localization process.

\subsection{Area Setup}

We model an indoor environment as a 2-D area with $L$ and $W$ denoting its length and width respectively. A set, $\mathcal{T}$, of passive RFID tags with known coordinates $\left(x_{t}, y_{t}\right), \forall t \in \mathcal{T}$ are placed on the floor of this area such that a grid of reference tags is formed with inter-tag spacing $\delta$. Within this area, a set, $\mathcal{N}$, of users with RFID reader-enabled terminals may be located and an accurate and fast estimation of their position, $\left(\widehat{x}_{u}, \widehat{y}_{u}\right), \forall u \in \mathcal{N}$, should be obtained. 


\subsection{Backscattered Path Loss Model and Read Range}

The communication link between the RFID components is half duplex, reader to tag and then tag to reader. In forward link, reader sends a modulated carrier to tags and powers up the tags. In return link, the tag receives the carrier for power supply and backscatters by changing the reflection coefficients of the antenna. In such a way, its $i d$ is sent to reader. The path loss of this two way link may be expressed as:

$$
P L(d)=P L_{o}+10 N \log \left(\frac{d}{d_{o}}\right)+X_{\sigma}(d B)
$$

where the variable $X_{\sigma}$, called the shadow fading, is a zero-mean Gaussian random variable in $\mathrm{dB}$ having a standard deviation of $\sigma_{d B}$ and is used to model the random nature of indoor signal propagation due to the effect of various environmental factors such as multipath, obstruction, orientation, etc. $N$ is the path loss exponent whose value depends on the frequency used.

$$
P L_{o}=G_{t} G_{r}\left(g_{t} \Gamma g_{r}\right)\left(\frac{\lambda}{4 \pi d_{o}}\right)^{4}
$$

is the path loss at reference distance $d_{o}$, where $G_{t}, g_{t}$, and $G_{r}, g_{r}$ are the gains of the reader and tag transmit and receive antennas, respectively. $\Gamma$ is a reflection coefficient of the tag and $\lambda$ the wavelength.

The combined forward and return path losses help calculate the maximum distance between the reader antenna and the tag at which the reader can decode the signals reflected from the tag, also called the read range and denoted as $R_{\max }$. For a desired reader, the received backscattered power, $P_{s}(d)$, is given by

$$
P_{s}(d)=\alpha_{B W} \Gamma P_{t} G_{t} G_{r} \times 10^{P L(d) / 10},
$$

where $P_{t}$ denotes the total transmit power, and $\alpha_{B W}$ is the spectrum power of a used channel normalized by the total power. In the absence of interference, the maximum read range a reader receiver can decode the backscattered signal is such that

$$
R_{\text {max }}=\arg \max _{d \geq 0} P_{s}(d) \geq T H,
$$

where a $T H$ represents a threshold value.

\subsection{Interference and Read Zone Reduction}

In subsection 3.2 the interference problem was described. Multiple reader-to-tag collisions, reader-to-tag collisions and interference from materials are the most implicating factors and therefore modelling them is essential.

Regarding multiple reader-to-tag collisions we utilize the following model. Let $R_{i}$ and $R_{j}$ denote the read ranges of readers $r_{i}$ and $r_{j}$ and $d_{i j}$ their distance. Apparently, if

$$
R_{i}+R_{j}>d_{i j}
$$


and $r_{i}$ and $r_{j}$ communicate at the same time, they will collide and the tags in the common area will not be detected. For characterizing the probability of simultaneous communication we assume that each reader is in a scanning mode with probability $p^{s c a n}$. Thus, the probability of collision, $P_{i j}^{C}$, between $r_{i}$ and $r_{j}$, if (5) is satisfied, depends on the probabilities $r_{i}$ and $r_{j}$ are in a scanning mode, $p_{i}^{s c a n}$ and $p_{j}^{\text {scan }}$, respectively, i.e. $P_{i j}^{C}=p_{i}^{\text {scan }} \times p_{j}^{\text {scan }}$.

Reader-to-reader collisions affect the $R_{\max }$ parameter. In (4) this factor had been neglected. However, when interfering readers exist, the actual interrogation range of the desired reader decreases to a circular region with radius $R_{\text {max }}^{I}$, which can be represented by

$$
R_{\max }^{I}=\arg \max _{d \in\left[0, R_{\max }\right]} S I R(d) \geq T H,
$$

where

$$
S I R(d)=\frac{P_{s}(d)}{\sum_{i} I_{i}}
$$

and $I_{i}$, interference from reader $r_{i}$.

Finally, for incorporating in the model problem of interference from materials, each reference tag $t$ is assigned a probability, $p_{t}$ of not being detected. Obviously high values of $p_{t}$ are assigned to tags which are mounted to such interfering materials.

\section{$5 \quad$ Positioning Methology}

\subsection{Architecture}

From architectural point of view, a location determination scheme can be either user-based or network-based. In the first case, each user is responsible for collecting and processing information necessary for determining his location, whereas, in the second case, a dedicated server is responsible for gathering all required data and finally providing the location estimates for all users. Processing capabilities, privacy and scalability issues are usually the main factors for selecting the appropriate approach. We propose a hybrid architecture as a compromise between them, i.e. both user and a central location server participate in the location decision process.

The reader of each user device queries for tags within its coverage in order to retrieve their $i d s$. A list of these retrieved $i d s$, called Tag-List, is forwarded to the Location Server. Based on these received Tag-List and a repository which correlates the $i d s$ of the reference tag with their location coordinates, the Location Server estimates the location of that user by employing an RFID-based positioning (see subsection 5.2) algorithm and finally returns this location estimate back to the corresponding user.

\subsection{Positioning Algorithms}

Let $\mathcal{D}_{u}$ denote the set of reference tags successfully detected from a user's reader $r_{u}$. Note that this happens if all factors described in 4.3 are considered. 
- Simple Average (SA)

This is the simplest scheme since it requires only $i d$ information of the detected reference tags. In this case, the user location is estimated as the simple average of the coordinates $\left(x_{t}, y_{t}\right)$ of all tags $t \in \mathcal{D}_{u}$, i.e.

$$
\left(\widehat{x}_{u}, \widehat{y}_{u}\right)=\left(\frac{\sum_{t \in \mathcal{D}_{u}} x_{t}}{\left|\mathcal{D}_{u}\right|}, \frac{\sum_{t \in \mathcal{D}_{u}} y_{t}}{\left|\mathcal{D}_{u}\right|}\right)
$$

- Weighted Average (WA)

Since some of the detected tags may be closer than others, biasing the simple averaging method is proposed as an alternative approach. This can be achieved by assigning a weight, $w_{t}$ to the coordinates of each tag $t \in \mathcal{D}_{u}$. These weights are based on their distance from the reader. Thus, (8) becomes

$$
\left(\widehat{x}_{u}, \widehat{y}_{u}\right)=\left(\frac{\sum_{t \in \mathcal{D}_{u}} w_{t} \cdot x_{t}}{\sum_{t \in \mathcal{D}_{u}} w_{t}}, \frac{\sum_{t \in \mathcal{D}_{u}} w_{t} \cdot y_{t}}{\sum_{t \in \mathcal{D}_{u}} w_{t}}\right)
$$

where $w_{t}=1 / \widehat{d}_{t}$ and $\widehat{d}_{t}$ is the estimated distance from the tag $t$ based on the level of the received signal strength.

- Multi-Lateration (ML)

Finally, a multi-lateration based approach is also investigated, according to which $\left(\widehat{x}_{u}, \widehat{y}_{u}\right)$ can be obtained by solving the following system of $\left|\mathcal{D}_{u}\right|$ equations:

$$
\left(x_{t}-x\right)^{2}+\left(y_{t}-y\right)^{2}=\widehat{d}_{t}, \quad \forall t \in \mathcal{D}_{u}
$$

Since $\widehat{d}_{t}$ are not accurate, the above system of equations can be solved by a standard LS approach as

$$
\left(\widehat{x}_{u}, \widehat{y}_{u}\right)=\left(A^{T} A\right)^{-1} A^{T} b
$$

where $A$ and $b$ are matrixes obtained after subtracting the last equation from the first $\left|\mathcal{D}_{u}\right|-1$ equations in the system of equations (10) for linearizing it, such that:

$$
\begin{gathered}
A_{t}=\left[2\left(x_{t}-x_{\left|\mathcal{D}_{u}\right|}\right) 2\left(y_{t}-y_{\left|\mathcal{D}_{u}\right|}\right)\right], \\
b_{t}=\left[x_{t}^{2}-x_{\left|\mathcal{D}_{u}\right|}^{2}+y_{t}^{2}-y_{\left|\mathcal{D}_{u}\right|}^{2}+\widehat{d}_{t}^{2}-\widehat{d}_{\left|\mathcal{D}_{u}\right|}^{2}\right], \\
\forall t \in \mathcal{D}_{u} \backslash\left\{\left|\mathcal{D}_{u}\right|\right\}
\end{gathered}
$$

\section{Simulation}

In this section we evaluate the performance of our approach through simulations, using Matlab, 18, as our simulation tool. As performance metric we use the mean location error (MLE), defined as the euclidean distance between the actual and the estimated position of a user. We provide and interpret results of the simulations we conducted for evaluating the influence of the parameters $\delta, \alpha$, and $R_{\max }$ in the system performance. In order to illustrate the performance 


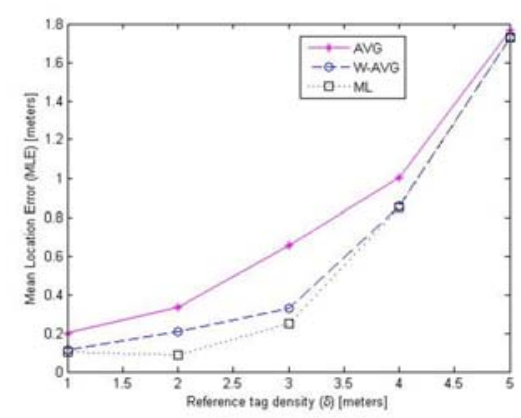

(a) Multi-user environment with $\beta=0$

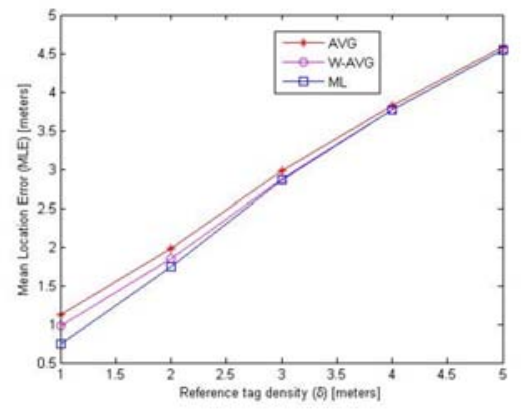

(b) Multi-user environment with $\beta=1$

Fig. 2. Impact of tag density $(\delta)$

degradation due to the collision problem and the essentiality of an anti-collision mechanism, we considered two multi-user environmental cases which differ in the level of the collision problem. Assuming that the probability user readers query their tags follows uniform distribution $U(\beta, 1)$, we set $\beta=0$ for the first case and $\beta=1$ for the second case. Apparently, for the second environment all users readers scan simultaneously for their tags and thus its performance is anticipated to be worse due to the collision problem among them.

\subsection{Impact of Tag Density}

Figures 2(a) and 2(b) illustrate the dependency of the $M L E$ on the tag density, $\delta$, when $\beta=0$ and $\beta=1$, respectively, and for the three rfid-based positioning methods described in subsection 5.2, i.e. $A V G, W-A V G$ and $L S$. For all cases, increasing the inter-tag spacing reduces the accuracy. However, when the collision problem is severe, the achieved accuracy and performance reduction are worse and thus a dense tag deployment is required for providing robustness. Finally, comparing the behavior of the three positioning schemes, we note that there is a benefit from the added complexity but in highly colliding environments the achieved benefit is not significant.

\subsection{Impact of Interference from Materials}

Similarly, figures $3(\mathrm{a})$ and $3(\mathrm{~b})$ show the impact of the level of interference $(\alpha)$ from environment materials for the two scenarios when $\delta=2$ meters. We observe that this factor increases the MLE, however, its impact is less severe compared to the parameter $\delta$.

\subsection{Impact of Interrogation Range}

Finally, in figures $4(\mathrm{a})$ and $4(\mathrm{~b})$ the influence of the maximum read range, $R_{\max }$, is depicted when $\delta=2$. For both scenarios we observe that when $R_{\max }=1$, 


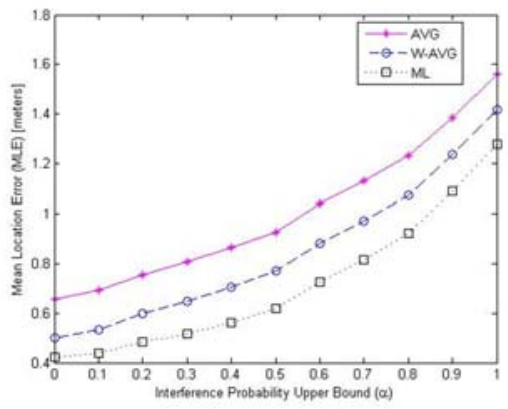

(a) Multi-user environment with $\beta=0$

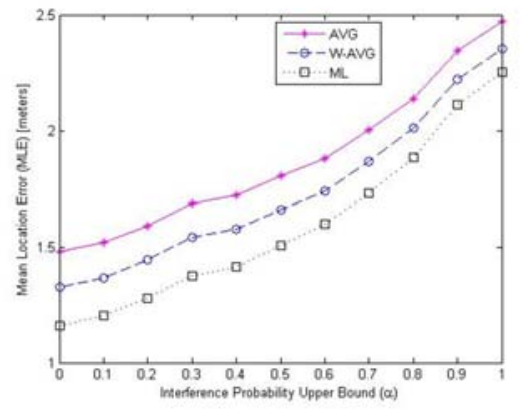

(b) Multi-user environment with $\beta=1$

Fig. 3. Impact of material interference level $(\alpha)$

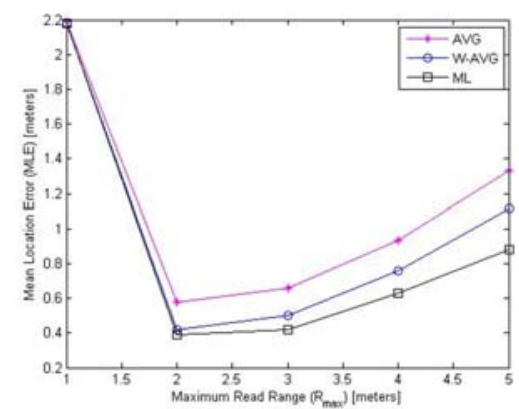

(a) Multi-user environment with $\beta=0$

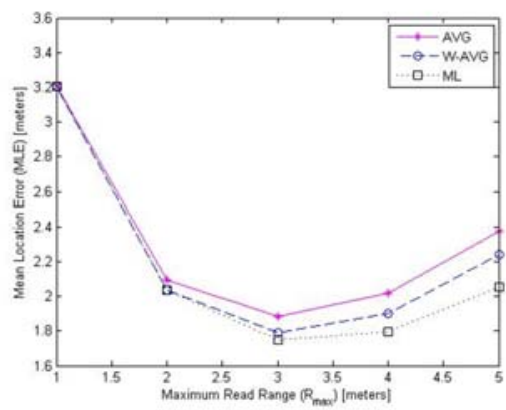

(b) Multi-user environment with $\beta=1$

Fig. 4. Impact of maximum read range $\left(R_{m} a x\right)$

the MLE is increased and this is because tags are not detected. When $\beta=0$, $R_{\max }=2$ gives the optimum performance for two main reasons; further than this collisions are more probable but also location information from far-away tags is included. For the second case, the optimum performance is achieved when $R_{\max }=3$ meters because of the collisions which prevents tags from being detected.

\section{Conclusion}

In this paper, we explore the applicability of the RFID technology in location sensing and the main design and environmental factors that should be considered before developing an RFID-based localization scheme. We focused on a scenario when the location of multiple reader-enabled terminals needs to be estimated based on the information retrieved from low cost passive tags, which are deployed in an area. We proposed a mathematical model for taking into account 
all implicating factors which affect the accuracy performance of the system, that is all types of collisions among its components, interference from materials, and temporal environmental changes. Extensive simulations were conducted to evaluate the impact of these parameters. More precisely, when reader collisions is not an issue, a low dense $(\delta \leq 4$ meters) deployment of passive tags can provide an accurate location information with error less than 1 meter. However, in a highly colliding environment, passive tags should be deployed with spacing of 1 meter in order to have similar location error resilience. Interesting remarks can be drawn regarding the communication range of readers. In the absence of collisions, short read range ( 2 meters) is beneficial. In contrast when readers attempt simultaneously access to the medium, a higher range (3-4 meters) results in better accuracy.

To summarize, RFID technology is suitable for positioning, but its performance degrades in highly populated environments and thus a denser tag deployment or/and a mechanism for controlling reader transmissions are required.

\section{References}

1. Kaplan, E.: Understanding GPS: Principles and Applications. Artech House (2005)

2. Hightower, J., Borrielo, G.: Location Systems for Ubiquitous Computing. IEEE Computer 34(8), 57-66 (2001)

3. Bahl, P., Padmanbhan, V.N.: RADAR: An In-Building RF-based User Location and Tracking System. In: IEEE Infocom, vol. 2, pp. 775-784 (2000)

4. Youssef, M., Agrawala, A.: The Horus Location Determination System. In: Mobisys, pp. 205-219 (2005)

5. King, T., Kopf, S., Haenselmann, T., Lubberger, C., Effelsberg, W.: COMPASS: a Probabilistic Indoor Positioning System Based on 802.11 and Digital Compasses. In: WinTeck, pp. 24-40 (2006)

6. Papapostolou, A., Chaouchi, H.: WIFE: Wireless Indoor positioning based on Fingerprint Evaluation. In: IFIP Networking (2009)

7. Want, R., Hopper, A., Falcao, V., Gibbons, J.: The Active Badge Location System. ACM Transactions on Information Systems 40(1), 91-102 (1992)

8. Priyyantha, N.B., Chakraborty, A., Balakrishnan, H.: The Cricket LocationSupport System. In: 6th International ACM MOBICOM (2000)

9. Want, R.: An introduction to RFID technology. IEEE Pervasive Computing 5(1), 25-33 (2006)

10. Ni, L.M., Liu, Y.: LANDMARK: Indoor Location Sensing Using Active RFID. Wireless Networks, 701-710 (2004)

11. Hightower, J., Want, R., Borriello, G.: SpotON: An indoor 3D location sensing technology based on RF signal strength. University of Washington, Department of Computer Science and Engineering, Seattle, WA, UW CSE 00-02-02 (February 2000)

12. Wang, C., Wu, H., Tzeng, N.-F.: RFID-based 3-D positioning schemes. In: IEEE INFOCOM, pp. 1235-1243 (2007)

13. Bouet, M., Pujolle, G.: A Range-Free 3-D Localization Method for RFID Tags Based on Virtual Landmarks. In: IEEE PIMRC (2008) 
14. Bekkali, A., Sanson, H., Matsumoto, M.: RFID Indoor Positioning Based on Probabilistic RFID Map and Kalman Filtering. In: Third IEEE International Conference on Wireless and Mobile Computing, Networking and Communications, WiMob 2007 (2007)

15. Yamanoi, K., Tanaka, K., Hirayma, M., Kondo, E., Kimuro, Y., Matsumototi, M.: Self-localization of Mobile Robots with WID System by using Support Vector Machine. In: Proceedings of 2004 IEEWRSI International Conference on Intelligent Robots and Systems, Sendai, Japan (2004)

16. Leong, K.S., Ng, M.L., Cole, P.H.: The reader collision problem in RFID systems. In: IEEE 2005 International Symposium on Microwave, Antenna, Propagation and EMC Technologies for Wireless Communications, Beijing, China, August 8-12 (2005)

17. http://www.epcglobalinc.org

18. http://www .mathworks.com 